\title{
Examination of the Vasa vasorum of the Human Great Saphenous Vein: A Scanning Electron Microscopy and 3D-Morphometry Study of Microvascular Corrosion Casts
}

\author{
M. Herbst ${ }^{1}$, T. Hölzenbein ${ }^{2}$ and B. Minnich ${ }^{1}$ \\ 1. Department of Cell Biology \& Physiology, Division of Animal Structure \& Function, Vascular \& \\ Exercise Biology Unit, University of Salzburg, Salzburg, Austria \\ 2. University clinics for vascular and endovascular surgery, PMU Salzburg, Salzburg, Austria
}

"Vasa vasorum" derives from Latin and literally means "vessels of the vessels", which describes their function of providing blood and oxygen to the arteries and veins vessel's wall. They, in turn, supply blood and oxygen to the rest of the body [1]. Hence the Vasa vasorum are a system of small blood vessels which supply large blood vessels. In detail a network of small arterioles, venules and capillaries which supply the outer two layers of large blood vessels $[2,3]$. The largest blood vessels in the body (e.g. the human great saphenous vein, the aorta, etc.) depend on this supporting network to maintain their health and function. Thus, the Vasa vasorum are an important part of the blood circulatory system [3, 4]. The structure of the Vasa vasorum varies first of all with the size as well as the function and location of the vessel. In the largest vessels it penetrates the tunica adventitia and the outer two third of the tunica media [5]. In smaller vessels the Vasa vasorum infiltrates only the adventitia. There are no Vasa vasorum in small vessels, because diffusion is sufficient for nourishment.

The aim of this study is the examination of the Vasa vasorum of the human great saphenous vein (Vena saphena magna, HGSV) in normal and pathological (varicose) conditions. We try to explore the optimality principles (minimal lumen volume, minimal pumping power, minimal lumen surface, minimal endothelial shear force) which underlie the design of this Vasa vasorum. Using vascular corrosion casting (VCC), scanning electron microscopy (SEM) and 3D-morphometry (M3) we are able to calculate optimum vessel diameter and branching indices of arterial, capillary and venous bifurcations and thus vascular optimality.

Another purpose of the study is the increase of knowledge of venous diseases, respectively to get an insight into the mechanism inducing varicogenesis. For these purposes we examined explanted segments of the HGSV which were taken during harvesting for coronary bypass grafts or varicose vein segments, from the University clinics for vascular and endovascular surgery, PMU Salzburg. The process of vascular corrosion casting starts with the dissection of the feeding artery from the surrounding tissue under a dissecting microscope, which is then cannulated with a glass cannula (gauge $\sim 80 \mu \mathrm{m}$ ).

Prior to the injection of the methacrylic resin "Mercox-Cl-2B", the Vasa vasorum have to be rinsed with saline using an automatic infusion pump (flow rate is about $7 \mathrm{ml} / \mathrm{hr}$ ). After polymerisation the specimens are macerated with $7.5 \%$ potassium hydroxide in order to remove all organic material. Thereafter, the casts are rinsed with distilled water for several times and frozen in distilled water. The ice-embedded casts are freeze-dried and mounted on a stub using the "conductive bridge method" [5]. After sputtering with gold and examination under the scanning electron microscope (FEI/Philips XL-30 ESEM), 3D-morphometry (M3) is performed.

Arterial feeders (Fig. 2) were found to approach the HGSV from nearby arteries every $15 \mathrm{~mm}$ forming a rich capillary network within the adventitia and the outer two thirds of the media in normal 
HGSV. In HGSV with intimal hyperplasia capillary meshes were found, which extended into the inner layers of the media. Within the media capillary meshes of the Vasa vasorum ran circularly (Fig. 2).

The diameters of (i) arterial VV ranged from 11.6 to $36.6 \mu \mathrm{m}$, (ii) capillary VV from 4.7 to $11.6 \mu \mathrm{m}$ and (iii) venous VV ranged from 11.6 to $200.3 \mu \mathrm{m}$ [6].

The three-dimensional network of the Vasa vasorum suggests that these layers are metabolically active and therefore require a continuous blood supply [6].

\section{References:}

[1] Ritman Erik L., Lerman A, Cardiovascurlar Research 75 (2007), p. 649-658.

[2] Minnich B. et al, Microscopy and Microanalysis 13 (2007), p. 488-489.

[3] Lametschwandtner A. et al, The Anatomical Record. Part A 281 (2004), p. 1372-1382.

[4] Kachlík D. et al, Vasa - Journal of Vascular Diseases 37 (2008), p. 127-136.

[5] Lametschwandtner A. et al, Mikroskopie 36, p. 270-273.

[6] Kachlík D. et al, Journal of Vascular Research 44 (2007), p. 157-166.

Figure 1. Abluminal view of the Vasa vasorum, which run predominately with the longitudinal axis of the vein. A part of the venous vessels is colored in blue (V) and some arterial vessels in red (A). Conductive bridges (CB) with colloidal silver.

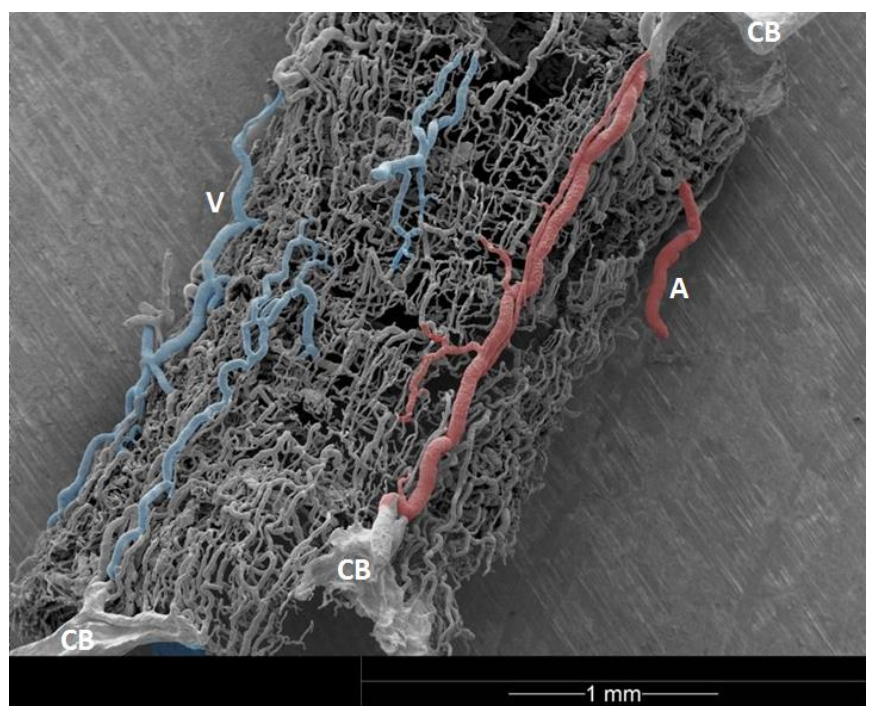

Figure 2. Luminal and abluminal view of the Vasa vasorum. The Vasa vasorum mainly run along the longitudinal axis (LA) of the vessel and radially to the muscle cells. Note the arterial feeder (AF) infiltrating this part of the HGSV. Conductive bridges (CB) with colloidal silver.

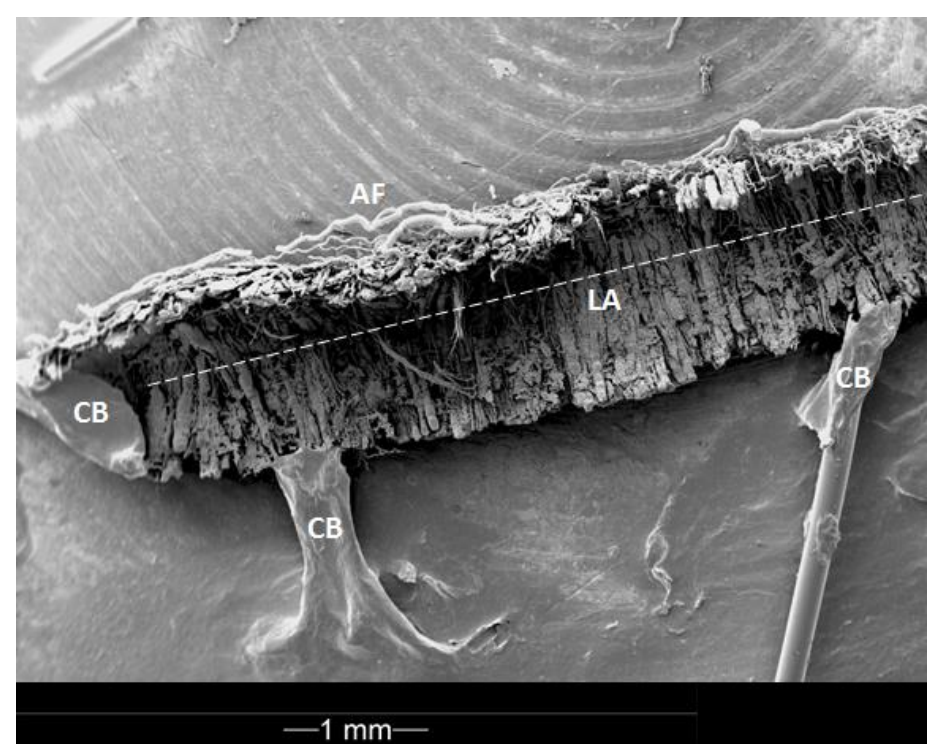

